University of Wollongong

Research Online

Faculty of Engineering and Information

Faculty of Engineering and Information

Sciences - Papers: Part B

Sciences

2018

\title{
Measurement accuracy of FBG used as a surface-bonded strain sensor installed by adhesive
}

Guangzhe xue

China University of Mining and Technology

Xinqiu Fang

China University of Mining and Technology

Xiukun Hu

China University of Mining and Technology

Libin Gong

University of Wollongong, lg283@uowmail.edu.au

Follow this and additional works at: https://ro.uow.edu.au/eispapers1

Part of the Engineering Commons, and the Science and Technology Studies Commons

Research Online is the open access institutional repository for the University of Wollongong. For further information contact the UOW Library: research-pubs@uow.edu.au 


\title{
Measurement accuracy of FBG used as a surface-bonded strain sensor installed by adhesive
}

\author{
Abstract \\ Material and dimensional properties of surface-bonded fiber Bragg gratings (FBGs) can distort strain \\ measurement, thereby lowering the measurement accuracy. To accurately assess measurement precision \\ and correct obtained strain, a new model, considering reinforcement effects on adhesive and measured \\ object, is proposed in this study, which is verified to be accurate enough by the numerical method. \\ Meanwhile, a theoretical strain correction factor is obtained, which is demonstrated to be significantly \\ sensitive to recoating material and bonding length, as suggested by numerical and experimental results. It \\ is also concluded that a short grating length as well as a thin but large-area (preferably covering the whole \\ FBG) adhesive can enhance the correction precision.

\section{Disciplines} \\ Engineering | Science and Technology Studies

\section{Publication Details} \\ Xue, G., Fang, X., Hu, X. \& Gong, L. (2018). Measurement accuracy of FBG used as a surface-bonded strain \\ sensor installed by adhesive. Applied Optics, 57 (11), 2939-2946.
}




\title{
Measurement accuracy of FBG used as surface-bonded strain sensor installed by adhesive
}

\author{
Guangzhe XUE, ${ }^{1}$ XINQIU Fang, ${ }^{1,}{ }^{*}$ XIUKUn Hu, ${ }^{1}$ LIBIN Gong ${ }^{2}$ \\ ${ }^{1}$ Key Laboratory of Deep Coal Resource Mining, Ministry of Education of China; School of Mines; China University of Mining \& Technology, \\ Xuzhou, 221116, China \\ ${ }^{2}$ School of civil, mining and environmental engineering, University of Wollongong, Wollongong, 2500, Australia \\ *Corresponding author: xinqiufang@cumt.edu.cn
}

Received 6 December 2017; revised 8 March, 2018; accepted XX Month XXXX; posted XX Month XXXX (Doc. ID XXXXX); published XX Month XXXX

Material and dimensional properties of surface-bonded FBGs can distort strain measurement, thereby lowering the measurement accuracy. To accurately assess measurement precision and correct obtained strain, a new model, considering reinforcement effects on adhesive and measured object, is proposed in this study, which is verified to be accurate enough by numerical method. Meanwhile, a theoretical strain correction factor is obtained, which is demonstrated to be significantly sensitive to recoating material and bonding length as suggested by numerical and experimental results. It is also concluded that short grating length as well as thin but large-area (preferably covering the whole FBG) adhesive can enhance the correction precision. (C) 2017 Optical Society of America

OCIS codes: (280.4788) Optical sensing and sensors; (060.3735) Fiber Bragg gratings; (130.6010) Sensors; (120.0120) Instrumentation, measurement, and metrology.

http://

\section{INTRODUCTION}

In recent decades, FBG sensors have been widely used in various fields. For instance, they can be used in intelligent materials [1-4] and structural health monitoring [5] to measure physical variables including stress, strain, temperature, velocity, and vibration. Their wide application can be attributed to their advantages over general electrical sensors, because they are favored by their light weight, small size, strong durability, high sensitivity and good immunity to electrometric interference and corrosion. Generally, there are two ways for FBGs to get the variation of strain, either by being embedded in the measured object to measure interior strain $[6,7]$ or by being directly bonded on the measured surface [8]. FBG, as a surface-bonded strain sensor, is expected to be perfectly integrated with the matrix, so that the strain in FBG should be equal to that on the surface of the host object. However, as both the soft recoating and the adhesive can significantly influence the strain transfer from the matrix to the fiber core, the strain in FBG is different from that on the surface of the matrix. Furthermore, when the measured object is soft, the original strain field on its surface can also be altered by pasted components, the so-called local reinforcement effect [9]. As a result, the strain measurement error is generated. In other words, regarding the strain in FBG, which can be obtained from the FBG sensing interrogator directly, as the true strain of host object without any modification can result in underestimation, therefore, the evaluation of strain measurement accuracy and essential correction of the strain obtained should be taken in practical application, especially in the measurement that requires high accuracy.

The accuracy and sensitivity of a directly bonded FBG strain sensor mainly depends on various specific factors of each surface-bonded FBG, including the bonding length [10], recoating and adhesive materials $[11,12]$, packing method [13-15] and even position of the FBG relative to the adhesive center [16]. The problem, known as "strain transfer", has been extensively studied for both embedded and surface-bonded FBGs [17-19]. Accordingly, measurement accuracy evaluation and error correction resulting from strain transfer can be implemented. However, unlike embedded FBG featured by centrosymmetric structure on the transverse surface, surface-bonded FBG is noncentrosymmetric as the FBG is fixed on only one side of the component. Thus, the strain in the surface-bonded FBG is transferred only through partial adhesive, rather than the total as in the centrosymmetric model for embedded FBG. In this context, the strain field is no longer centrosymmetric, which enables it to be perfectly expressed by the shear-lag model of Cox [20]. A largely intractable problem occurs if the effect of FBG on the strain field within adhesive and the effect of adhesive on host components' strains, referred to as "reinforcement effects" [21], could not be generalized exactly. The former one can be recognized as "global reinforcement effect", as the cross-section of adhesive is not so big that the FBG perturbs the strain field within the whole adhesive [22]. Meanwhile, the latter one can be regarded as "local reinforcement effect", because the measured objects are generally big enough. As a result, some additional assumptions for these two reinforcement effects should be made. As for the assumptions concerning adhesive structures, half-spindle liked adhesives (Fig. 1) have been simplified as a rectangle containing fibers [23], a rectangle below fibers [24], and a ring outside the fibers [25] respectively, for example, as shown in Fig. 2. Among them, the ring outside fibers enables the embedded FBG model to be easily quoted to represent the strain transfer process within surface-bonded FBG. 
Unfortunately, different assumptions concerning adhesive structures can reach different analytical solutions, because it is not easy to theoretically define the participating portion of the adhesive in the strain transfer [26]. The theoretical strain transfer coefficients obtained by these models may be not so exact, and errors can also come from negligence of the reinforcement effect on the adhesive. What's more, when the host matrix is soft, the reinforcement effect on it can be significant enough to introduce sizable estimate deviation, which makes the strain transfer coefficient obtained fail to improve strain measurement accuracy of FBG. Nevertheless, these problems have been barely reported in the literatures.

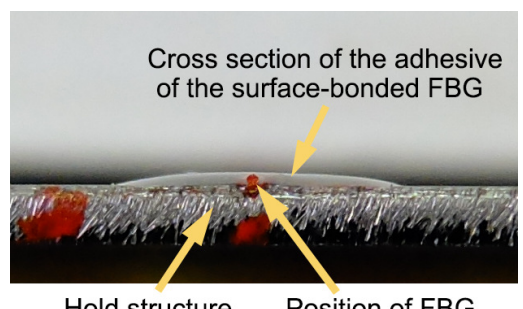

Fig. 1. Photography of the transverse section of the surface-bonded FBG.

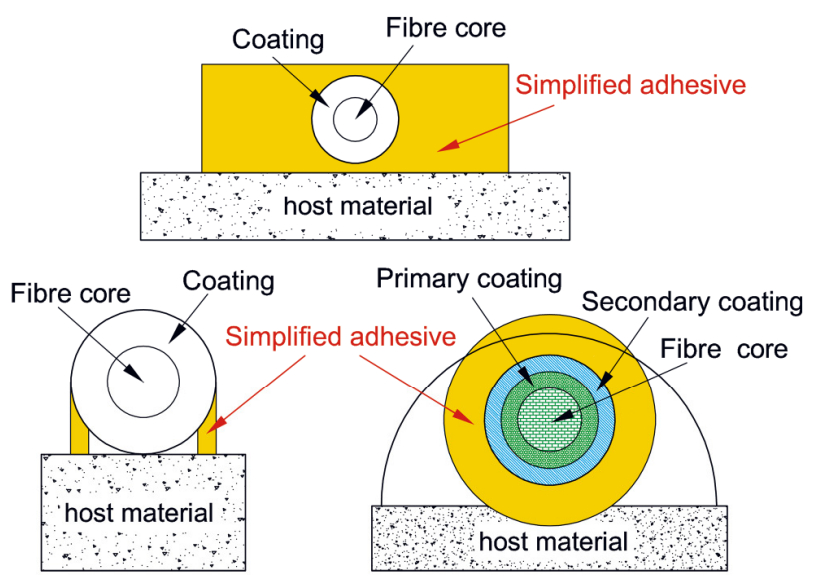

Fig. 2. Simplifications of adhesive in surface-bonded FBG model as rectangle containing FBG, rectangle below FBG and ring outside the FBG respectively.

Taking the reinforcement effects on both adhesive and measured component into account, a new model for surface-bonded FBG is proposed in this paper. Through theoretical and numerical analysis, the model is demonstrated to be able to achieve a precise expression of the strain in FBG and a realistic strain correction factor, which is dominated by properties and dimensions of recoating and adhesive materials. The sensitivity of the factor, which reveals potential harms to accuracy, is analyzed, and corresponding suggestions to ensure its accuracy in practice are proposed. Finally, influences of bonding length and recoating material to the correction factor precision are evaluated by experiments.

\section{BASIC ASSUMPTIONS}

It is expected that the FBG should be bonded well and closely to the measured surface to achieve perfect strain transfer. In practice, following procedure for sticking FBG is employed to ensure the bonding quality: after being cleaned, the measured surface is coated with moderate epoxy, and then the FBG placed in the adhesive is closely bonded to the measured surface by tweezers; after that, the adhesive is pressed slightly with plastic film covering it; finally, it is solidified in room temperature for two days. By following this procedure, possible air bubble and gap can be eliminated, and thus good bond between FBG, epoxy and measured surface can be achieved. In this analysis, the adhesive simplification as rectangular which contains FBG, which resembles the shape of adhesive in Fig. 1, is employed, base on which, a new model is established to assess the strain measured by FBG, as shown in Fig. 3. The subscripts $f, c, a$ and $m$ present fiber core, protective recoating, adhesive layer and matrix, respectively; $E, G, \mu, r, \varepsilon, \sigma$ and $\tau$ are Young's modulus, shear modulus, Poisson's ratio, radius, axial strain, axial stress and shear stress, respectively; the parameters $L, w, h_{0}, h_{\mathrm{b}}$ and $h_{\mathrm{t}}$ are half bonding length, adhesive width, total adhesive thickness, adhesive bottom thickness and adhesive top thickness, respectively. Based on assumptions of perfectly bonded interfaces, linear elasticity of all components' behaviours and exclusive suffering of shear deformation [23-25], this model assumes the impacts of reinforcement effects and certain adhesive participating in the strain transfer as follows:

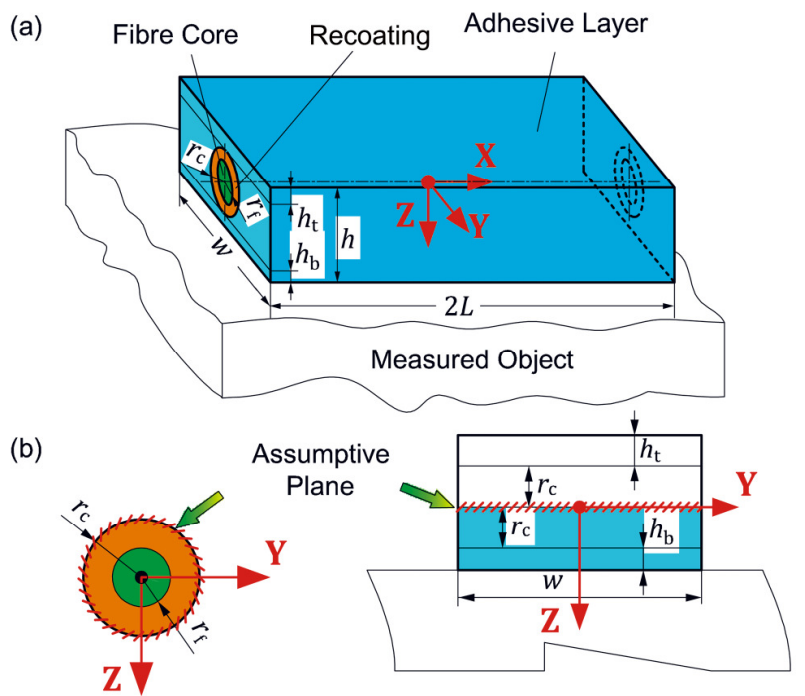

Fig. 3. (a) Three-dimensional diagrammatic sketch of simplified adhesive layer. (b) Cross-section review of the improved analytical model for surface-bonded FBG.

(a)

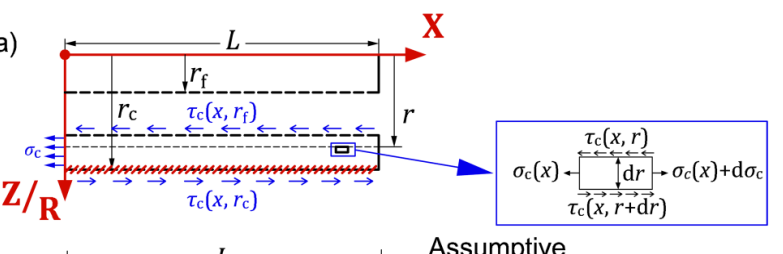

(b)

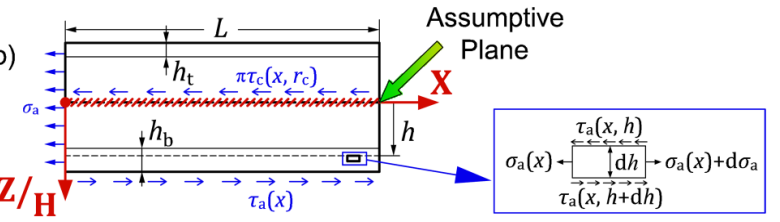

Fig. 4. (a) Stress distribution and infinitesimal element diagram in protective recoating. (b) Stress distribution and infinitesimal element diagram in adhesive layer. 
(1) Considering the two parts of stress transfer processes, namely the strain transfers through protective recoating and adhesive, an assumptive plane is supposed to exist within adhesive to be the link. The lower adhesive (Fig. 3 (b)) transfers stress, over whole width $w$, while the upper part deforms passively. The position of the assumptive plane is dependent on the location of FBG, and it is assumed to be in the geometrical center of the FBG in this model.

(2) The structure of underlying adhesive is thought to be under the action of a pair of anti-plane shear stresses. One is on the assumptive plane from protective recoating, and the other is on the bottom surface from bonded component, as shown in Fig. 4. Considering local reinforcement effect, the former shear stress from protective recoating is modified by the factor $\pi$, which comes from the fact that the anti-plane shear stress distributed on the outside surface of cylindrical recoating with the perimeter of $2 \pi r_{c}$ is concentrated on the corresponding projected area on the assumptive plane with a width of only $2 r_{\mathrm{c}}$.

(3) As measured objects are much larger than adhesive layer and FBG, considering global reinforcement effect, the bonded surface is considered to be homogeneous isotropic elastic semi space under the action of anti-plane shear stress from adhesive layer.

\section{STRAIN MEASUREMENT ACCURACY ANALYSIS OF SURFACE-BONDED FBG}

Strain measurement accuracy can be estimated by analytical strain transfer coefficient, and disturbances by temperature [27] are not discussed in this paper. During the strain transfer process in recoating, as shown in Fig. 4 (a), the shear stress inside recoating $\tau_{\mathrm{c}}(x, r)$ can be expressed as a function of $r$ :

$$
\tau_{\mathrm{c}}(x, r)=\frac{r_{\mathrm{f}}^{2} E_{\mathrm{f}}}{2 r}\left(-\frac{\mathrm{d} \varepsilon_{\mathrm{f}}}{\mathrm{d} x}-\frac{\left(r^{2}-r_{\mathrm{f}}^{2}\right)}{r_{\mathrm{f}}^{2}} \frac{E_{\mathrm{c}}}{E_{\mathrm{f}}} \frac{\mathrm{d} \varepsilon_{\mathrm{c}}}{\mathrm{d} x}\right) .
$$

During the strain transfer process in adhesive, as shown in Fig. 4 (b), the shear stress within the underlying adhesive, considering local reinforcement effects, is a function of $h$ :

$$
\begin{aligned}
& \tau_{\mathrm{a}}(x, h)=-\frac{r_{\mathrm{f}}^{2} E_{\mathrm{f}}}{2 r_{\mathrm{c}}} \\
& \quad \times\left[\pi \frac{\mathrm{d} \varepsilon_{\mathrm{f}}}{\mathrm{d} x}+\frac{\pi\left(r_{\mathrm{c}}^{2}-r_{\mathrm{f}}^{2}\right)}{r_{\mathrm{f}}^{2}} \frac{E_{\mathrm{c}}}{E_{\mathrm{f}}} \frac{\mathrm{d} \varepsilon_{\mathrm{c}}}{\mathrm{d} x}+\frac{2 r_{\mathrm{c}}\left(h+r_{\mathrm{c}}+h_{\mathrm{t}}\right)}{r_{\mathrm{f}}^{2}} \frac{E_{\mathrm{a}}}{E_{\mathrm{f}}} \frac{\mathrm{d} \varepsilon_{\mathrm{a}}}{\mathrm{d} x}\right] .
\end{aligned}
$$

The protective recoating, adhesive and fiber core together constitute the strain transfer medium, within which strains are continuous and on the same order of magnitude. As the strain gradient is equal, the error induced by conversions as follows is considered to be insignificant:

$$
\begin{aligned}
& \frac{E_{\mathrm{c}}}{E_{\mathrm{f}}} \frac{\mathrm{d} \varepsilon_{\mathrm{c}}}{\mathrm{d} x} \rightarrow \frac{E_{\mathrm{c}}}{E_{\mathrm{f}}} \frac{\mathrm{d} \varepsilon_{\mathrm{f}}}{\mathrm{d} x} . \\
& \frac{E_{\mathrm{a}}}{E_{\mathrm{f}}} \frac{\mathrm{d} \varepsilon_{\mathrm{a}}}{\mathrm{d} x} \rightarrow \frac{E_{\mathrm{a}}}{E_{\mathrm{f}}} \frac{\mathrm{d} \varepsilon_{\mathrm{f}}}{\mathrm{d} x} .
\end{aligned}
$$

Then Eqs. (1) and (2) can be converted to the following formulae respectively:

$$
\tau_{\mathrm{c}}(x, r)=-\left(\frac{r_{\mathrm{f}}^{2} E_{\mathrm{f}}}{2 r}+\frac{\left(r^{2}-r_{\mathrm{f}}^{2}\right) E_{\mathrm{c}}}{2 r}\right) \frac{\mathrm{d} \varepsilon_{\mathrm{f}}(x)}{\mathrm{d} x} .
$$

$$
\tau_{\mathrm{a}}(x, h)=-\left[\frac{\pi r_{\mathrm{f}}^{2} E_{\mathrm{f}}}{2 r_{\mathrm{c}}}+\frac{\pi\left(r_{\mathrm{c}}^{2}-r_{\mathrm{f}}^{2}\right) E_{\mathrm{c}}}{2 r_{\mathrm{c}}}+\left(h+r_{\mathrm{c}}+h_{\mathrm{t}}\right) E_{\mathrm{a}}\right] \frac{\mathrm{d} \varepsilon_{\mathrm{f}}(x)}{\mathrm{d} x}
$$

By integral of shear strains in protective recoating and adhesive layer, the relationship between axial strain in fiber core $\varepsilon_{\mathrm{f}}(x)$ and that on the bottom surface of adhesive $\varepsilon_{\mathrm{a}}(x)$ is acquired:

$$
\frac{\mathrm{d}^{2} \varepsilon_{\mathrm{f}}(x)}{\mathrm{d} x^{2}}-\alpha^{2} \varepsilon_{\mathrm{f}}(x)=-\alpha^{2} \varepsilon_{\mathrm{a}}(x)
$$

where $\alpha$ presents the numerical relation between the strain in FBG and that in adhesive, and it is a parameter that reflects influence of the shear-lag effect in a surface-bonded FBG, in which impacts of mechanical and geometrical properties of protective recoating and adhesive are involved.

$$
\begin{gathered}
\alpha=\left\{\begin{array}{c}
\frac{r_{\mathrm{f}}^{2}\left(E_{\mathrm{f}}-E_{\mathrm{c}}\right) \ln \left(\frac{r_{\mathrm{c}}}{r_{\mathrm{f}}}\right)}{2 G_{\mathrm{c}}}+\frac{\pi\left(h_{\mathrm{b}}+r_{\mathrm{c}}\right)\left[r_{\mathrm{f}}^{2} E_{\mathrm{f}}+\left(r_{\mathrm{c}}^{2}-r_{\mathrm{f}}^{2}\right) E_{\mathrm{c}}\right]}{2 r_{\mathrm{c}} G_{\mathrm{a}}} \\
\left.+\left(1+\mu_{\mathrm{a}}\right)\left[h_{0}^{2}-\left(h_{\mathrm{t}}+r_{\mathrm{c}}\right)^{2}\right]+\frac{1}{2}\left(1+\mu_{\mathrm{c}}\right)\left(r_{\mathrm{c}}^{2}-r_{\mathrm{f}}^{2}\right)\right\}^{-1 / 2} .
\end{array}\right.
\end{gathered}
$$

As both the stress and strain transmissions on the free-end planes of the bonded fiber are equal to zero, the boundary condition is given by $\varepsilon_{\mathrm{f}}(L)=\varepsilon_{\mathrm{f}}(-L)=0$, and the relation between the strain in FBG, $\varepsilon_{\mathrm{f}}(x)$, and corresponding strain on bottom surface of adhesive, $\varepsilon_{\mathrm{a}}(x)$, can be further calculated and expressed as

$$
\varepsilon_{\mathrm{f}}(x)=\varepsilon_{\mathrm{a}}(x)\left[1-\frac{\cosh (\alpha x)}{\cosh (\alpha L)}\right] .
$$

Then, the anti-plane stress applied on the measured surface can be expressed as

$$
\tau_{\mathrm{a}}(x)=\frac{\alpha E A \sinh (\alpha x)}{w \cosh (\alpha L)} \varepsilon_{\mathrm{a}}(x) .
$$

where $E A=\pi r_{\mathrm{f}}^{2} E_{\mathrm{f}}+\pi\left(r_{\mathrm{c}}^{2}-r_{\mathrm{f}}^{2}\right) E_{\mathrm{c}}+w h_{0} E_{\mathrm{a}}$, representing the equivalent stiffness of the entirety comprising those of the adhesive, recoating and fiber core.

Absolutely, the strain on the bonded area of measured object is the same as that on the bottom surface of adhesive, and it can be computed by subtracting the increment of strain generated by the anti-plane stress from the true strain $\varepsilon_{\mathrm{m}}$, which is the unaffected strain when FBG has not been pasted.

$$
\begin{aligned}
& \varepsilon_{\mathrm{m}}- \varepsilon_{\mathrm{a}}(x)=\frac{\left(1+\mu_{\mathrm{m}}\right) \varepsilon_{\mathrm{a}}(x)}{\pi E_{\mathrm{m}}} \\
& \times \int_{0}^{L} \int_{-\frac{w}{2}}^{\frac{w}{2}}\left[\frac{\left(3 \mu_{\mathrm{m}}-1\right) x}{\left(x^{2}+y^{2}\right)^{1.5}}-\frac{3 \mu_{\mathrm{m}} x^{3}}{\left(x^{2}+y^{2}\right)^{2.5}}\right] \frac{\alpha E A \sinh (\alpha x)}{w \cosh (\alpha L)} \mathrm{d} y \mathrm{~d} x .
\end{aligned}
$$

Then, the conversion is obtained 
$\varepsilon_{\mathrm{a}}(x)=\varepsilon_{\mathrm{m}}\left\{1+\frac{2 \alpha E A\left(1+\mu_{\mathrm{m}}\right)}{\pi E_{\mathrm{m}} \cosh (\alpha L)} \int_{0}^{L} \frac{\sinh (\alpha x)\left[\left(\mu_{\mathrm{m}}-1\right) w^{2}-4 x^{2}\right]}{x\left(4 x^{2}+w^{2}\right)^{1.5}} \mathrm{~d} x\right\}$.

There is a linear correlation between the strain on the bonded area and the true strain of measured object. The factor $\beta$ is defined to express the reinforcement effect on pasted object, which is determined by all the components of the surface-bonded FBG:

$$
\beta=1+\frac{2 \alpha E A\left(1+\mu_{\mathrm{m}}\right)}{\pi E_{\mathrm{m}} \cosh (\alpha L)} \int_{0}^{L} \frac{\sinh (\alpha x)\left[\left(\mu_{\mathrm{m}}-1\right) w^{2}-4 x^{2}\right]}{x\left(4 x^{2}+w^{2}\right)^{1.5}} \mathrm{~d} x .
$$

As the axial strain within FBG, no matter inserted or surface-bonded, is unevenly distributed and what the FBG sensing interrogator identifies is the central wavelength of reflected spectrum, this paper regards the average strain within the heartland of FBG with the range of half its length as the equivalent strain, which can be calculated by the FBG sensing interrogator in practice. Then, the strain measured by $\mathrm{FBG} \varepsilon_{\mathrm{FBG}}$ can be expressed as

$$
\varepsilon_{\mathrm{FBG}}=\frac{2 \int_{0}^{L_{\mathrm{f}} / 2} \varepsilon_{\mathrm{f}}(x) \mathrm{d} x}{L_{\mathrm{f}}}=\varepsilon_{\mathrm{m}} \beta\left[1-\frac{2 \sinh \left(\alpha L_{\mathrm{f}} / 2\right)}{\alpha L_{\mathrm{f}} \cosh (\alpha L)}\right] .
$$

The ratio of measured strain $\varepsilon_{\mathrm{FBG}}$ to the true strain of measured object $\varepsilon_{\mathrm{m}}$ is defined as the measured strain proportion $\eta_{\mathrm{FBG}}$ to indicate the strain measurement accuracy, which is given as

$$
\eta_{\mathrm{FBG}}=\beta-\frac{2 \beta \sinh \left(\alpha L_{\mathrm{f}} / 2\right)}{\alpha L_{\mathrm{f}} \cosh (\alpha L)} .
$$

The strain correction factor can be obtained as

$$
k=\frac{1}{\eta_{\mathrm{FBG}}}=\frac{\alpha L_{\mathrm{f}} \cosh (\alpha L)}{\beta\left[\alpha L_{\mathrm{f}} \cosh (\alpha L)-2 \sinh \left(\alpha L_{\mathrm{f}} / 2\right)\right]} .
$$

The accuracy of strain correction factor $k$ depends on the amount of reinforcements effects, which include the strain decrease on measured object (dramatically significant when the measured object is soft) and the strain distortion within adhesive layer, as clearly illustrated in the 3D numerical model in Fig. 5. This model is established in software ANSYS Workbench based on the transverse contour of surface-bonded FBG in Fig. 1. Parameters employed are listed in Table 1. To ensure the symmetry from a practical point of view, FBG is typically positioned in the geometrical center of the adhesive layer, in both $\mathrm{X}$ and $\mathrm{Y}$ directions. The cloud pictures reveal the three-dimensional strain reduction within the pasted object, and display non-uniform noncentrosymmetric strain distributions in adhesive layer and protective recoating. In addition, the FBG influences the strain field in the whole cross-section within adhesive, while the adhesive only disturbs the strain field near it.

Table 1. Parameters Employed in Numerical Model ${ }^{a}$

\begin{tabular}{ccccc}
\hline Components & $\boldsymbol{E} \mathbf{( M P a )}$ & $\boldsymbol{\mu}$ & $\boldsymbol{r}(\mathbf{m m})$ & $\mathbf{2 L} \mathbf{( m m )}$ \\
\hline Fiber core & 7200 & 0.17 & 0.0625 & 24 \\
Recoating a & 17 & 0.48 & 0.125 & 23 \\
Adhesive layer & 4000 & 0.34 & $2 \times 0.65$ & 20 \\
Measured object & 6000 & 0.3 & - & -
\end{tabular}

a Values are given by the manufacturer of FBG employed in the section 5 and the measured object is as soft as polyimide.
In Fig. 6, the theoretical axial strain distributions within FBG are in good agreement with simulation results within the scope of the FBG. The theoretical strain within FBG in this paper is more close to simulation results than previous study by Wan et al. [25], which verifies higher precision of theoretical formulas and more realistic strain correction factor $k$ in this paper. Moreover, the theoretical strain ratio on measured surface, factor $\beta$, is equal to the numerical one within the length of FBG, and it is clear that the reinforcement effect on measured surface is well expressed as well.

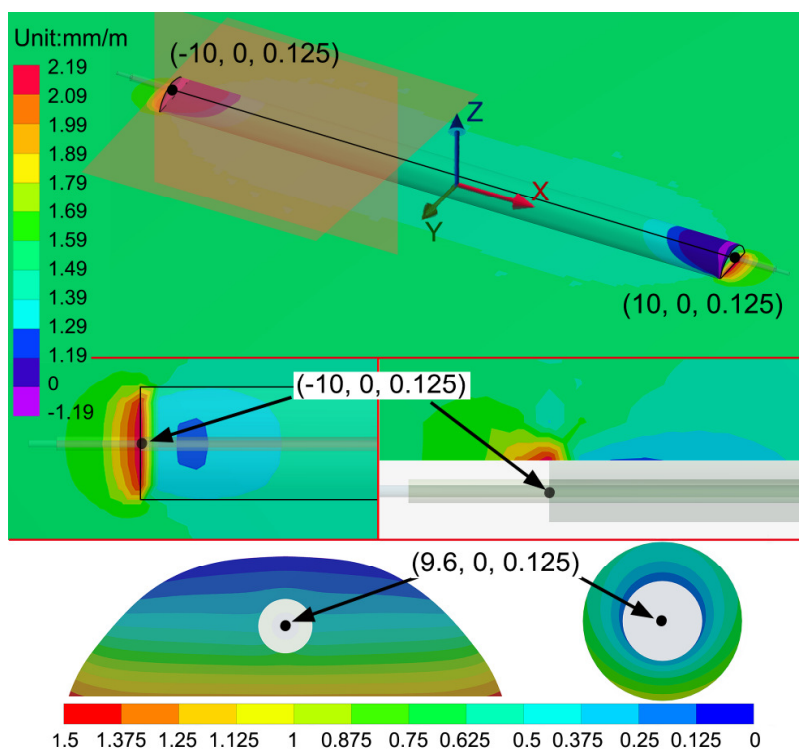

Fig. 5. Strain field in the numerical model under the true strain of $0.15 \%$ with top and bottom adhesive thicknesses of $0.2 \mathrm{~mm}$. Strain disturbances caused by local reinforcement effects on measured object at vertical and longitudinal view and non-centrosymmetric strain fields within adhesive and protective recoating at the plane $\mathrm{X}=9.6 \mathrm{~mm}$ are also illustrated clearly.

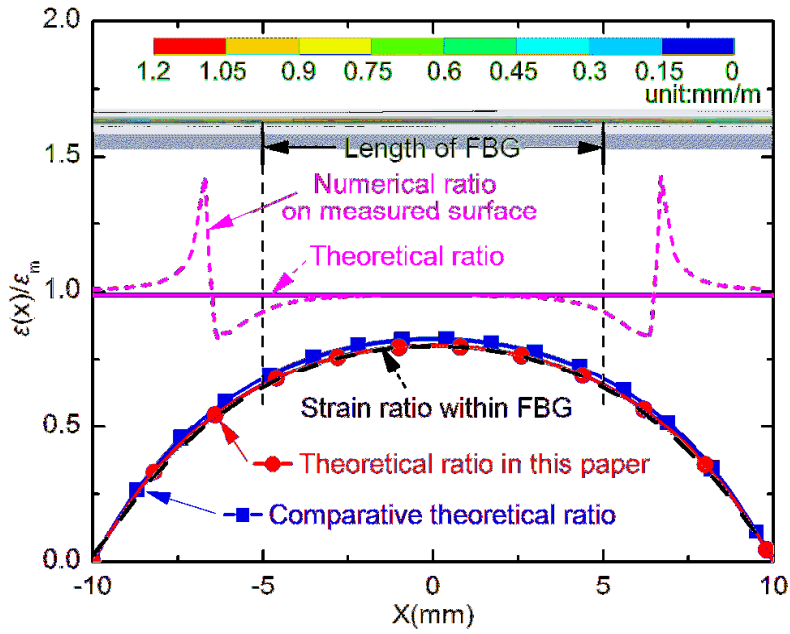

Fig. 6. Normalized strain distribution along the measured surface and within FBG obtained by numerical method (dotted line) and theoretical formulas (solid line) under the true strain of $0.15 \%$. Theoretical solutions in this paper (with round dot) achieve higher accuracy than the contrast one [21] (with square). 


\section{NUMERICAL INVESTIGATION FOR THE STRAIN CORRECTION FACTOR}

As can be seen in Eq. (16), the strain correction factor $k$ is dominated by geometrical and mechanical parameters of all components of surface-bonded FBG. Both precise theoretical strain correction factor $k$ and exactly measured characteristic parameters are crucial to achieve high correction accuracy. However measurement error is inevitable in practice so that it is essential to make sure that the sensitivity of $k$ to these parameters can help reduce the debasement of correction accuracy by these errors. In this section, the numerical model proposed in Section 3 is employed, in which all the materials are assumed to be isotropic and linearly elastic. Adopted variables are as shown in Table 2, in which their original values and ranges are listed. Assuming the length of FBG as $10 \mathrm{~mm}$, Figs. 7 to 10 display the factor $k$ versus these primary influential parameters, in which the relative error (ratio of the difference between theoretical and numerical results to numerical results) is also graphed to indicate accuracy.

\section{Table 2. Value Ranges of Variables}

\begin{tabular}{cccccc}
\hline Variables & $\begin{array}{c}\boldsymbol{E}_{\mathbf{c}} \\
(\mathbf{M P a})\end{array}$ & $\begin{array}{c}\mathbf{2 L} \\
(\mathbf{m m})\end{array}$ & $\begin{array}{c}\boldsymbol{w} \\
(\mathbf{m m})\end{array}$ & $\begin{array}{c}\boldsymbol{h}_{\mathbf{t}} \\
(\mathbf{m m})\end{array}$ & $\begin{array}{c}\boldsymbol{h}_{\mathbf{b}} \\
(\mathbf{m m})\end{array}$ \\
\hline Original values & $2 / 20 / 50$ & 20 & 2 & 0 & 0 \\
Ranges & $2-300$ & $10-50$ & $0.3-5$ & $0-1$ & $0-1$ \\
\hline
\end{tabular}

Apparently, variations of the strain correction factor $k$ obtain by Eq. (16) are extremely similar to those obtained by simulation. Particularly, the strain correction factor $k$ is significantly sensitive to Young's modulus of protective recoating as well as bonding length, especially when they are small, as shown in Figs. 7 and 8. It means that a small measurement error in practice for these two parameters may greatly distort the value of $k$. Moreover, when these two parameters are small, the relative error, which is called systematic error, will be large as well. It is clear that strain correction precision is closely related to these critical parameters, namely recoating material properties and bonding length. As the bonding length is generally limited in practice, a certain bonding length $(2 L>15 \mathrm{~mm})$ and a stiff protecting recoating $\left(E_{\mathrm{c}}\right.$ $>20 \mathrm{MPa}$ ) are suggested to be employed to achieve a high accuracy in strain measurement and correction.

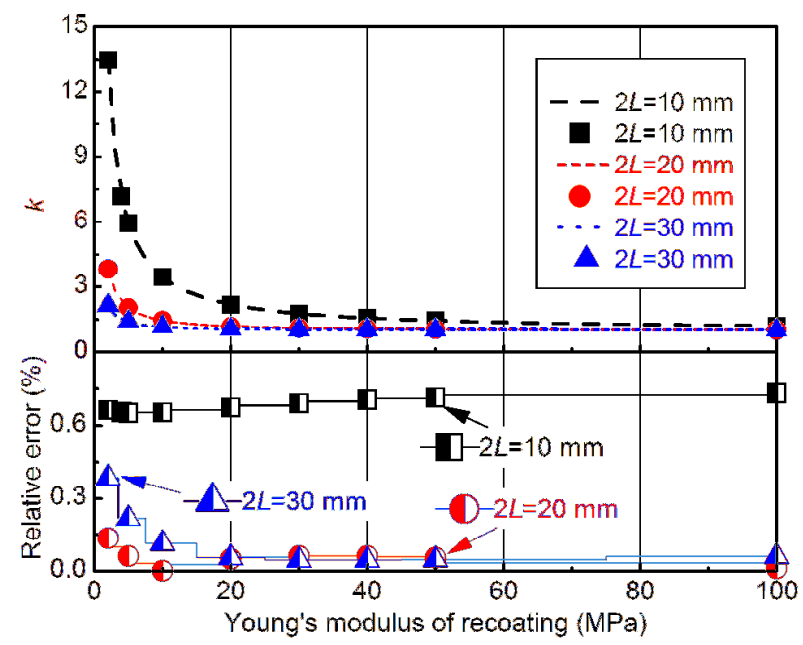

Fig. 7. Evolution of theoretical strain factor $k$ and its relative error with Young's modulus of protective recoating for bonding lengths of 10, 20 and $30 \mathrm{~mm}$.

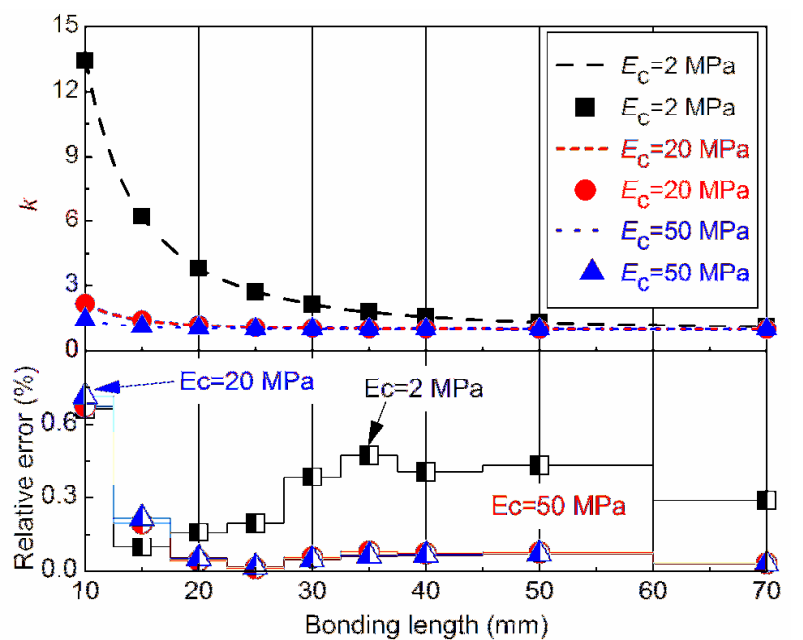

Fig. 8. Evolution of theoretical strain factor $k$ and its relative error with bonding length for the protective recoating's Young's modulus of 2, 20 and $50 \mathrm{MPa}$.

In contrast, the other geometric parameters of adhesive demonstrate less influence on the sensitivity of theoretical strain correction factor $k$ and its accuracy, as shown in Figs. 9 and 10. Factor $k$ and its relative error increase slightly with the width and thickness of the adhesive. Owing to the reinforcement effect, larger stiffness of adhesive generally induces more strain disturbances on the measured surface. Therefore, although Eq. (16) offers a high strain correction precision, a thin and slim adhesive is still suggested. What's more, considering the rapidly increasing relative error of factor $k$ by the bottom thickness of adhesive as shown in Fig. 10, FBG should cling to the measured surface with adhesive covered in practice, and the bonding procedure mentioned in Section 2 is recommended.

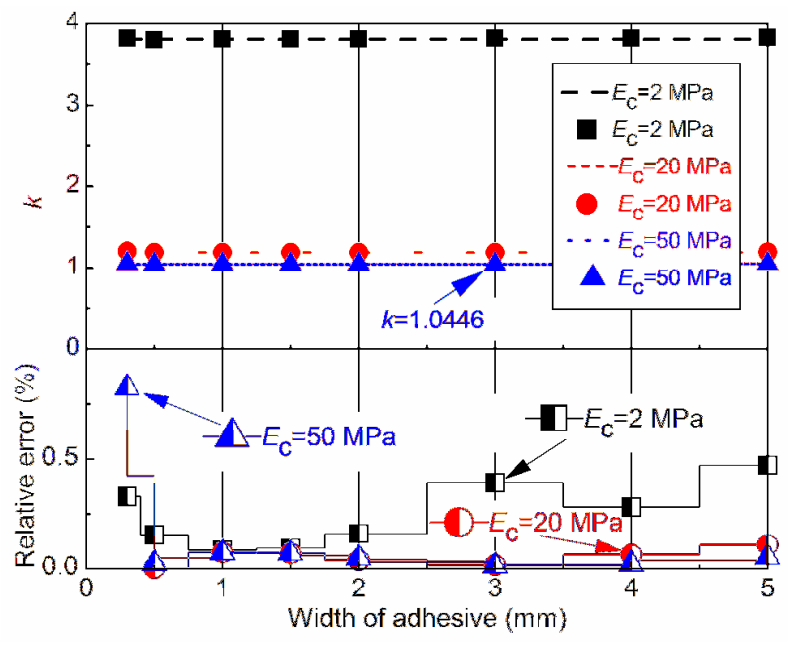

Fig. 9. Factor $k$ and its relative error versus width of adhesive for the protective recoating's Young's modulus of 2, 20 and $50 \mathrm{MPa}$. 


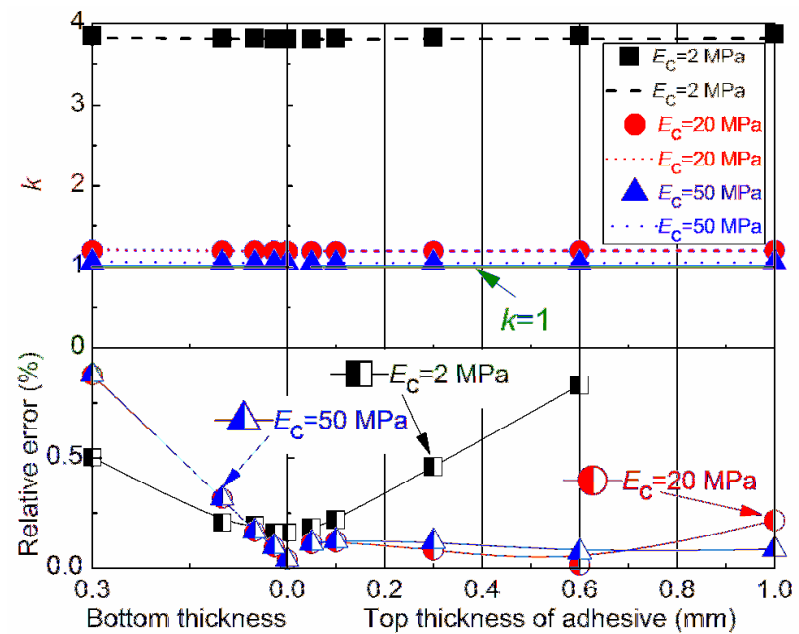

Fig. 10. Factor $k$ and its relative error versus thickness of adhesive for the protective recoating's Young's modulus of 2,20 and $50 \mathrm{MPa}$. Bottom thickness (left); top thickness (right).

\section{EXPERIMENTAL VALIDATION}

To validate the accuracy of factor $k$ obtained by Eq. (16) as well as its sensitivity to dimensions of adhesive layer, two experiments are carried out, as shown in Fig. 11. FBGs are bonded axially onto the test specimens in uniaxial tests on the MTS Criterion TM Universal Testing System. These FBGs employed, are recoated with acrylate (Young's Modulus of $17 \mathrm{MPa}$ ) with a 0.25 -mm radius. Uniaxial tension tests are implemented in bolts in coal mine in experiment I, and uniaxial compression tests are conducted in sandstone specimens collected from underground mine in experiment II. Resistive strain gauges are also bonded in the same places for comparisons. In Experiment I, the surfaces of bolts are polished, on which two FBGs with different grating lengths are bonded to suffer tension; and in Experiment II, an FBG is pasted on the surface of sandstone specimen to bear compression. The optimized bonding procedure mentioned in Section 2 is followed, and samples are cured at room temperature for 2 days. The epoxy, named Ausbond EP05, is adopted, with the Young's modulus of $\sim 4 \mathrm{GPa}$ (provided by manufacturer). Three FBGs without recoating are also employed as comparison in Experiment II.

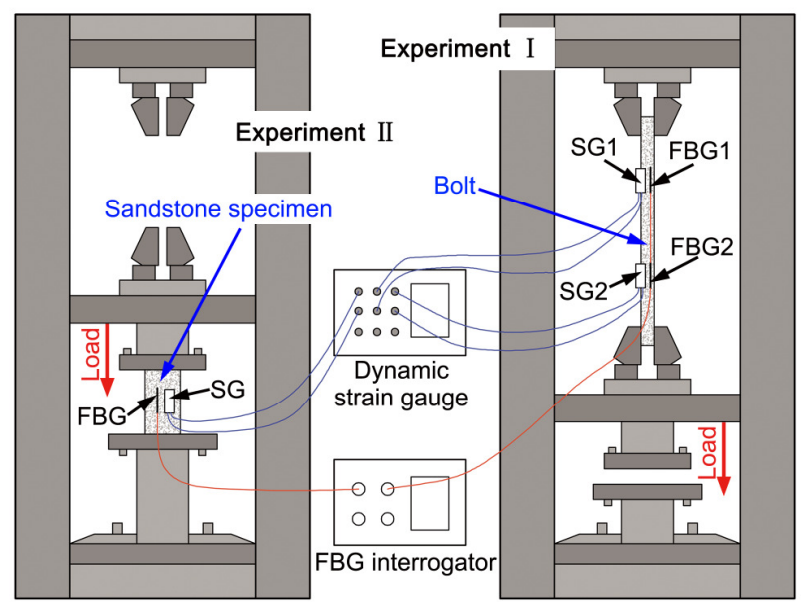

Fig. 11. Schematic diagrams of the two experiments. In this, SG is short for the strain gauge.
Tables 3 and 4 show the values of strain correction factor obtained by experiments, recorded as $k_{\mathrm{e}}$, and that calculated by Eq. (16), recorded as $k_{\mathrm{t}}$, as well as their relative errors $(\delta k)$ for various bonding lengths and adhesive widths. Apparently, results obtained by experiments are in good agreement with those in Section 4. First, factor $k$ is demonstrated to decrease with the increase in bonding length, as shown in Fig. 8, indicating increasingly effective strain transfer. A smaller decrement of $k$ is also showed as the bonding length increases. Similarly, relative errors of theoretical results also decrease with bonding length. Considering the high sensitivity to short bonding length, relative errors in experiments are much larger than those in simulation when the bonding length is less than $16 \mathrm{~mm}$. But as the errors are below $3 \%$, the theoretical factor $k$ in this paper is proved to be accurate and workable to correct the measured strain by FBG surface installed by adhesive.

Table 3. Strain Correction Factor $\boldsymbol{k}$ Attained by Experimental Measurement and Theoretical Prediction (Eq. 16) in Experiment I. Young's Modulus of Tested Bolts is Measured to Be 2.76e2 GPa.

\begin{tabular}{ccccccc}
\hline No. & $\begin{array}{c}\mathbf{2} \mathbf{L} \\
(\mathbf{m m})\end{array}$ & $\begin{array}{c}\mathbf{2 L} \\
(\mathbf{m m})\end{array}$ & $\begin{array}{c}\boldsymbol{w} \\
(\mathbf{m m})\end{array}$ & $\boldsymbol{k} \mathbf{e}$ & $\boldsymbol{k} \mathbf{t}$ & $\begin{array}{c}\boldsymbol{\delta} \boldsymbol{k} \\
(\mathbf{\%})\end{array}$ \\
\hline \multirow{2}{*}{$\mathrm{I}-1$} & 10 & 16.01 & 3.01 & 1.3966 & 1.4368 & 2.874 \\
& 15 & 16.35 & 3.22 & 1.4061 & 1.4582 & 3.705 \\
$\mathrm{I}-2$ & 10 & 20.02 & 2.85 & 1.2519 & 1.2350 & 1.350 \\
& 15 & 20.11 & 3.05 & 1.2482 & 1.2538 & 0.449 \\
$\mathrm{I}-3$ & 10 & 23.23 & 3.15 & 1.1543 & 1.1494 & 0.425 \\
& 15 & 23.36 & 3.08 & 1.1480 & 1.1596 & 1.009 \\
$\mathrm{I}-4$ & 10 & 27.35 & 3.24 & 1.0978 & 1.0864 & 1.043 \\
& 15 & 27.68 & 3.57 & 1.0887 & 1.0894 & 0.065 \\
$\mathrm{I}-5$ & 10 & 31.40 & 3.09 & 1.0592 & 1.0514 & 0.736 \\
& 15 & 31.07 & 3.26 & 1.0636 & 1.0579 & 0.540 \\
\hline
\end{tabular}

Table 4. Strain Transfer Coefficients of FBGs Attained by Experimental Measurement and Theoretical Prediction (Eq. 16) in Experiment II. Young's Modulus of Tested Sandstone is Measured to Be 27.5 GPa.

\begin{tabular}{|c|c|c|c|c|c|}
\hline No. & $\begin{array}{c}2 L \\
(\mathrm{~mm})\end{array}$ & $\begin{array}{c}w \\
(\mathrm{~mm})\end{array}$ & $k_{\mathbf{e}}$ & $k_{\mathrm{t}}$ & $\begin{array}{c}\delta k \\
(\%)\end{array}$ \\
\hline II- 1 a & 16.43 & 6.15 & 0.9878 & 1.0009 & 1.266 \\
\hline II-2 & 16.32 & 6.33 & 1.3682 & 1.4163 & 3.521 \\
\hline II-3 a & 27.01 & 5.85 & 0.9839 & 1.0004 & 1.683 \\
\hline II-4 & 27.68 & 4.92 & 1.0801 & 1.0839 & 0.348 \\
\hline II- 5 a & 34.75 & 4.54 & 0.9963 & 1.0003 & 0.397 \\
\hline II-6 & 35.03 & 4.47 & 1.0361 & 1.0333 & 0.265 \\
\hline
\end{tabular}

a The strain sensors employed were bare FBGs which are unrecoated, and their theoretical values are obtained considering $r_{\mathrm{c}}$ as $0.625 \mathrm{~mm}\left(r_{\mathrm{f}}\right)$.

From another perspective, FBGs with long grating length deviate more in strain correction. Their spectra probably suffer degeneration like the FBG2 in Experiment II-2 in Fig. 12, reflected by insensitivity and widened bandwidth. With the strain increasing, serious mutation of the spectrum resulting from the large deformation can cause damage to the precision of strain correction. Furthermore, FBGs without recoating are of high accuracy and could sense almost the entire strain, only suffering little local reinforcement effect on the measured surface, shown in Table 4. They are not considered to be used as strain sensors because they are fragile and their thin glass cores without protective materials can be easily fractured. In fact, only FBGs with short grating length are suitable for point strain measurement, and the model developed in this paper is also valid for them. 


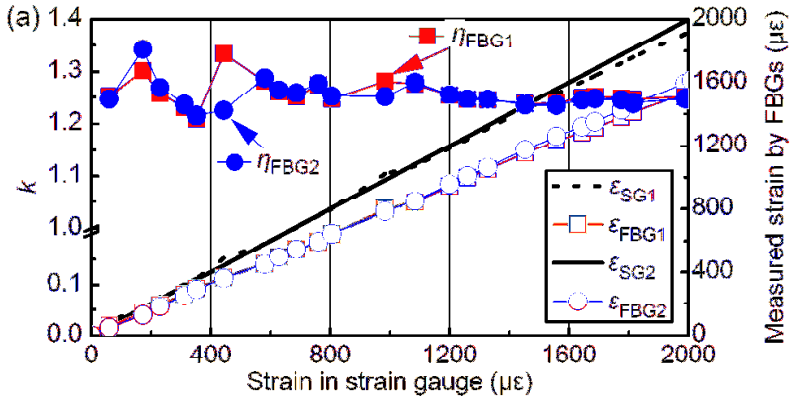

(b)

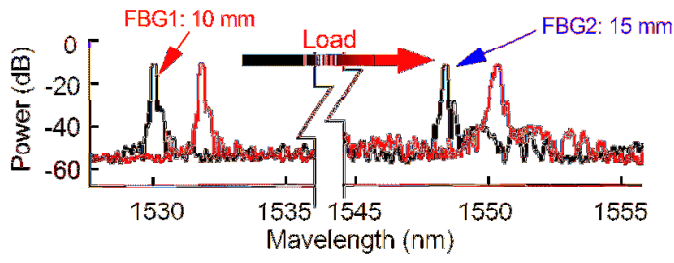

Fig. 12. (a) Strain curves and correction factor $k$ measured in Experiment I-2 by FBGs. (b) Spectra of the FBGs at the strain in SG2 of $2010 \mu \varepsilon$.

\section{CONCLUSIONS}

In this study, the strain measurement accuracy and correction factor of FBG used as surface-bonded sensor are studied by proposing a new theoretical model. Taking the reinforcement effects into consideration, the model is demonstrated to be more accurate by numerical analysis. Sensitivity of the strain correction factor to five primary parameters is fully discussed, which suggests the short bonding length and soft recoating material to be the most critical two factors. These two factors are verified by experiment to lower the correction precision in practice due to possible dimension measurement errors. Accordingly, an FBG with short grating length as well as thin but large-area (preferably covering the whole FBG) adhesive is suggested to enhance the correction accuracy in practice. This study offers practical but precise correction for the surface-bonded FBG installed by adhesive to infer the true strain within the measured object.

Funding Information. Fundamental Research Funds for the Central Universities (No. 2014ZDPY22)

\section{REFERENCES}

1. Z. Y. Gao, X. J. Zhu, Y. B. Fang and H. S. Zhang, "Active monitoring and vibration control of smart structure aircraft based on FBG sensors and PZT actuators," Aerosp. Sci. Technol. 63, 101-109 (2017).

2. R. Morsy, H. Marzouk, M. Haddara and X. Gu, "Multi-channel random decrement smart sensing system for concrete bridge girders damage location identification," Eng. Struct. 143, 469-476 (2017).

3. A. Vieira, R. D. Oliveira, O. Frazão, J. M. Baptista and A. T. Marques, "Effect of the recoating and the length on fiber Bragg grating sensors embedded in polymer composites," Mater. Des. 30, 1818-1821 (2009).

4. D. Lo Presti, C. Massaroni, D. Formica, P. Saccomandi, F. Giurazza, M. A. Caponero and E. Schena, "Smart Textile Based on 12 Fiber Bragg Gratings Array for Vital Signs Monitoring," IEEE Sens. J. 17, 6037-6043 (2017).

5. K. Al Handawi, N. Vahdati, P. Rostron, L. Lawand and O. Shiryayev, "Strain based FBG sensor for real-time corrosion rate monitoring in pre-stressed structures," Sens. Actuators, B 236, 276-285 (2016).

6. V. P. Matveenko, I. N. Shardakov, A. A. Voronkov, N. A. Kosheleva, D. S. Lobanov, G. S. Serovaev, E. M. Spaskova and G. S. Shipunov, "Measurement of strains by optical fiber Bragg grating sensors embedded into polymer composite material," Struct. Control Health Monit. 25, e2118,(2018).

7. R. Marsili, G. Rossi, and E.Speranzini, "Fibre Bragg Gratings for the Monitoring of Wooden Structures," Materials, ma11010007 (2017).

8. Xi. W. Ye, Y. H. Su and P. S. Xi, "Statistical Analysis of Stress Signals from Bridge Monitoring by FBG System," Sensors, s18020491, (2018).

9. A. Ajovalasit and B. Zuccarello, "Local reinforcement effect of a strain gauge installation on low modulus materials," J. Strain Analysis, 40, 643653 (2005).

10. S. C. Her and C. Y. Huang, "The Effects of Adhesive and Bonding Length on the Strain Transfer of Optical Fiber Sensors," Appl. Sci. 6, app6010013 (2016).

11. W. Zhang, W. M. Chen, Y. J. Shu, X. H. Lei and X. M. Liu, "Effects of bonding layer on the available strain measuring range of fiber Bragg gratings," Appl. Opt. 53, 885-891 (2014).

12. H. Li, L. Q. Zhu, M. L. Dong, X. P. Lou and Y. K. Guo, "Analysis on strain transfer of surface-bonding FBG on Al 7075-T6 alloy host," Optik 127, 1233-1236 (2016).

13. B. Zuccarello, L. D'Acquisto and G. Di Franco, "Analysis of the accuracy of fiber-optic strain transducers installed by using composite smart patches," J. Strain Anal. Eng. Des. 50, 373-385 (2015).

14. Y. M. Zhang, L. Q. Zhu, F. Luo, M. L. Dong, R. T. Yang, W. He and X.P. Lou, "Comparison of Metal-Packaged and Adhesive-Packaged Fiber Bragg Grating Sensors," IEEE Sens. J. 16, 5958-5963 (2016).

15. C. S. Song, J. X. Zhang, M. Yang, J.G. Zhang and W. Yuan, "Strain transfer error experiments and analysis on CFRP laminates using FBG sensors," in proceedings of the 2 nd International Conference on Advanced Materials, Mechanical and Structural Engineering , S. H. Hong, J. Seo \& K. Moon ed. (CRC, 2015), pp. 315-320.

16. K. T. Wan, "Quantitative Sensitivity Analysis of Surface Attached Optical Fiber Strain Sensor," IEEE Sens. J. 14, 1805-1812 (2014).

17. F. Ansari and L. Yuan, "Mechanics of bond and interface shear transfer in optical fiber sensors," J. Eng. Mech. 124, 385-394 (1998).

18. D. S. Li, H. N. Li, L. Ren and G. B. Song, "Strain transferring analysis of fiber Bragg grating sensors," Opt. Eng. 45, 024402 (2006).

19. W.Y. Li, C.C. Cheng, Y.L Lo, "Investigation of strain transmission of surfacebonded FBGs used as strain sensors," Sens. Actuators A 149 (2009) 201207.

20. H. L. Cox, "The elasticity and strength of paper and other fibrous materials," Br. J. Appl. Phys. 3, 72-79 (1952).

21. A. Ajovalasit and B.Zuccarello, "Local reinforcement effect of a strain gauge installation on low modulus materials," J. Strain Anal. Eng. Des. 40, 643-653 (2005).

22. S. Zike and L. P. Mikkelsen, "Correction of Gauge Factor for Strain Gauges Used in Polymer Composite Testing," Exp. Mech. 54, 393-403 (2014).

23. H. T. Zhao, Q. B. Wang, Y. Qiu, J. A. Chen, Y. Y. Wang and Z. M. Fan, "Strain transfer of surface-bonded fiber Bragg grating sensors for airship envelope structural health monitoring," J. Zhejiang Univ.-Sci. A. 13, 538545 (2012).

24. S. C. Her and C. Y. Huang, "Effect of coating on the strain transfer of optical fiber sensors," Sensors 11, 6926-6941 (2011).

25. K. T. Wan, C. K. Y. Leung and N. G. Olson, "Investigation of the strain transfer for surface-attached optical fiber strain sensors," Smart Mater. Struct. 17, 035037 (2008).

26. C. C. Perry, "The resistance strain gage revisited," Expl. Mech. 24, 286299 (1984).

27. M. Waris, P. J. Liotier, S. Drapier, "Effect of the mold on the residual strain field monitored with optical fibers sensors in resin transfer molding processes," J. Compos. Mater. 48, 2589-2601 (2014). 